\title{
Body Composition and Physical Fitness in a Cohort of US Military Medical Students
}

\author{
Sarah D. Mitchell, BA, Richard Eide, BA, Cara H. Olsen, DrPH, and \\ Mark B. Stephens, MD
}

Medical school requires that students balance academic schedules with other lifestyle demands, including nutrition, physical fitness, and wellness. We retrospectively reviewed trends in body composition and physical fitness of a cohort of military medical students attending the Uniformed Services University. Although students were able to maintain muscular endurance and body composition, cardiorespiratory endurance, as measured by a timed 1.5-mile run, declined significantly over a 2 -yr period. ( $\mathrm{J}$ Am Board Fam Med 2008;21:165-167.)

The rigors of education are stressful for many medical students, and the manifestations of this stress can be positive or negative. Common maladaptive responses to stress include physical inactivity and poor nutrition. This is reflected by weight gain and declining fitness levels. This not only has deleterious effects on physical well-being: stress-induced neglect of proper nutrition and lack of sufficient physical activity are detrimental to medical students' mental health as well. ${ }^{1}$

Previous studies have assessed medical students' health using questionnaires about healthy behaviors $^{2}$ or self-assessments of individual student's health status. ${ }^{3}$ Most students seem to be in compliance with current exercise recommendations when they enter medical school. ${ }^{4}$ What happens to their levels of fitness during medical school, however, has never been formally measured. To our knowledge, no study has ever directly measured the

This article was externally peer reviewed.

Submitted 19 August 2007; revised 17 October 2007; accepted 24 October 2007.

From Walter Reed Army Medical Center, Washington, DC (SDM); Uniformed Services University, Bethesda, MD (RE, CHO, MBS).

Funding: Intramural Uniformed Services University Protocol HU81BY.

Conflict of interest: none declared.

Disclaimer: The views expressed herein are those of the authors. They do not represent official policy of the Department of Defense, the Department of the Army, or the Department of the Navy.

Corresponding author: Mark B. Stephens, MD, Associate Professor of Family Medicine, Uniformed Services University, Bethesda, MD 20814 (E-mail: mstephens@usuhs.mil). longitudinal fitness of students through the course of medical school.

The purpose of our study was to describe changes in body mass index (BMI) and physical fitness parameters of medical students at the Uniformed Services University as they progressed through medical school. At this university, all students are on active military duty and are required to maintain a minimum level of fitness within prescribed military standards. We hypothesized, therefore, that student fitness levels would remain constant throughout medical school.

\section{Methods}

After receiving approval from the institutional ethics committee, we retrospectively reviewed physical fitness parameters of a cohort of 163 military medical students enrolled at the Uniformed Services University in Bethesda, Maryland. Each student was on active duty in the US Navy at the time of the study. As part of personnel standards, the US Navy requires satisfactory performance on a biannual physical readiness test (PRT). Elements of the Navy PRT include: (1) measurements of height and weight; (2) the maximum number of push-ups performed in a 2-minute period; (3) the maximum number of sit-ups performed in a 2 -minute period; and (4) a timed 1.5-mile run. These component tests are used as surrogate measures of body composition (height and weight); muscular endurance (sit-ups and push-ups); and cardiorespiratory endurance (1.5-mile run). The validity and reliability 
Table 1. Mean Physical Fitness Scores for Medical Students from Autumn 2004 through Autumn 2006

\begin{tabular}{lcccc}
\hline Semester & $\begin{array}{c}\text { Sit-ups } \\
(\mathrm{n})\end{array}$ & $\begin{array}{c}\text { Push-ups } \\
(\mathrm{n})\end{array}$ & $\begin{array}{c}\text { BMI } \\
\left(\mathrm{kg} / \mathrm{m}^{2}\right)\end{array}$ & $\begin{array}{c}\text { 1.5-mile Run Time } \\
(\mathrm{min}: \mathrm{sec})\end{array}$ \\
\hline Autumn 2004 & 90 & 61 & 24.2 & $10: 54$ \\
Spring 2005 & 93 & 60 & 24.2 & $11: 17$ \\
Autumn 2005 & 87 & 57 & 24.2 & $11: 29$ \\
Spring 2006 & 90 & 61 & 24.2 & $11: 22$ \\
Autumn 2006 & 89 & 61 & 24.2 & $11: 31$ \\
$P$ (linear trend) & .68 & .11 & .91 & $<.001$ \\
\hline
\end{tabular}

BMI, body mass index.

of each of these tests as longitudinal measures of physical fitness has previously been established. ${ }^{5}$

Each student is required to complete the PRT twice every year. Beginning with the freshman class in the autumn of 2004 and ending in the autumn of 2006, we tracked PRT scores longitudinally through time using an existing database that records PRT scores for all Navy personnel. As each subsequent class matriculated, their scores were added to the database. Individual data were recorded for height, weight, sit-ups, push-ups and 1.5 mile run time. Data were de-identified, transferred to a spreadsheet, and imported into SPSS software (v.12.0; SPSS Inc., Chicago, IL) for statistical analysis.

Our study was adequately powered to detect a $5 \%$ change in body mass index and a $2 \%$ change in 1.5 -mile run times during the students' time in medical school. Differences in physical fitness scores were determined using a mixed-model analysis of variance for repeated measures followed by post hoc tests for linear trends over time. Basic descriptive statistics were used for all other analyses.

\section{Results}

Student BMI measurements did not appreciably change during the first 3 years of medical school. The average BMI was 24.2 at each of the 5 time points $(P=.91)$. The number of push-ups and sit-ups that students performed also remained constant. The number of push-ups completed was between 57 and 61 at each test $(P=.11)$. The average number of sit-ups was 90 in the autumn of 2004 and 89 in the autumn of $2006(P=.68)$ (Table 1$)$. Notably, the times for the 1.5 -mile run significantly increased as students progressed through medical school. Average run time increased from
10:54 in the autumn of 2004 to 11:31 in the autumn of $2006(P<.001)$. Overall, students showed an absolute decrease of $4 \%$ in cardiorespiratory fitness as measured by their run times.

\section{Conclusion}

To our knowledge, ours is the first study to describe longitudinal physical fitness measures in a cohort of medical students as they progress through medical school. Our students were able to maintain their BMI and muscular endurance during the first 3 years of medical school. Their cardiorespiratory fitness, however, declined significantly. In fact, this decline far exceeded the anticipated age-related sedentary change of $0.1 \%$ to $0.3 \%$ per year in young adults. ${ }^{6}$ It also far exceeds the decline in fitness of Navy personnel in general. ${ }^{7}$ The reasons for this are unclear. Minimum PRT standards are published (www.navy-prt. com) so people can target their exercise and eating habits in the weeks preceding the physical readiness test simply to achieve a "passing score." Whether the score on the PRT test reflects a students' usual $\mathrm{BMI}$, therefore, is debatable.

There are several important limitations to our study. Our students are slightly older (average age at matriculation, 25 years) and we have more men (69\%) than national medical school averages. To our knowledge, no other school requires their students to meet physical performance standards. This potentially limits the generalizability of our results. It is notable, however, that cardiorespiratory fitness declined despite the school's requirement that students meet minimum fitness standards. Therefore, it is reasonable to hypothesize that declines in fitness would be even greater in medical students at schools without a similar fitness requirement.

Encouraging personal health and wellness improves medical students' ability to promote the health of their patients. ${ }^{2}$ Patients have greater confidence in the health advice received from physicians they perceive to be healthy. ${ }^{8,9}$ Developing and maintaining healthy lifestyle habits during medical school, therefore, has clinical implications that extend far beyond the educational years.

\section{References}

1. Kjeldstadli K, Tyssen R, Finset A, et al. Life satisfaction and resilience in medical school a six-year longitudinal, nationwide and comparative study. BMC Med Educ 2006;6:48. 
2. Frank E, Hedgecock J, Elon LK. Personal health promotion at US medical schools: a quantitative study and qualitative description of deans' and students' perceptions. BMC Med Educ 2004;4:29.

3. Liang MTC, Dombrowski HT, Allen TW, et al. Do medical students' knowledge and attitudes about health and exercise affect their physical fitness? J Am Osteopath Assoc 1993;93:1020-4, 1028-32.

4. Frank E, Galuska DA, Elon LK, Wright EH. Personal and clinical exercise-related attitudes and behaviors of freshmen US medical students. Res Q Exerc Sport 2004;75:112-21.

5. Larsen GE, George JD, Alexander JL, Fellingham GW, Aldana SG, Parcell AC. Prediction of maxi- mum oxygen consumption from walking, jogging, or running. Res Q Exerc Sport 2002;73:66-72.

6. Stewart KJ. Physical activity and aging. Ann N Y Acad Sci 2005;1055:193-206.

7. Trent LK, Hurtado SL. Longitudinal trends and gender differences in physical fitness and lifestyle factors in career US Navy personnel (1983-1994). Mil Med 1998;163:398-407.

8. Hash RB, Munna RK, Vogel RL, Bason JJ. Does physician weight affect perception of health advice? Prev Med 2003;36:41-4.

9. Frank E, Breyan J, Elon L. Physician disclosure of healthy personal behaviors improves credibility and ability to motivate. Arch Fam Med 2000;9:287-90. 